\title{
Genetic variants of the endothelial NO synthase gene (eNOS) may confer increased risk of sporadic congenital heart disease
}

\author{
K. Zhou ${ }^{1 *}$, Y. Wang ${ }^{2 *}$, W. Peng ${ }^{3}$, J. Sun ${ }^{3}$, Y.M. Qing ${ }^{1}$ and X.M. Mo ${ }^{3}$ \\ ${ }^{1}$ Department of Cardiology, \\ The Affiliated Children's Hospital of Nanjing Medical University, \\ Nanjing, China \\ ${ }^{2}$ Department of Neonatology, \\ The Affiliated Children's Hospital of Soochow University, Soochow, China \\ ${ }^{3}$ Department of Cardiothoracic Surgery, \\ The Affiliated Children's Hospital of Nanjing Medical University, \\ Nanjing, China \\ *These authors contributed equally to this study. \\ Corresponding author: X.M. Mo \\ E-mail: wy4tc@sina.com
}

Genet. Mol. Res. 13 (2): 3805-3811 (2014)

Received April 15, 2013

Accepted October 29, 2013

Published May 16, 2014

DOI http://dx.doi.org/10.4238/2014.May.16.4

\begin{abstract}
The endothelial NO synthase (eNOS) enzyme is expressed during the early stages of cardiogenesis and plays an important role in normal heart development. Genetic variations of eNOS G894T have been shown to influence individual susceptibility to some phenotypes of congenital heart disease (CHD) in different populations. We conducted a case-control study comprised of 945 CHD patients and 972 non-CHD individuals in a Chinese population. Two functional single nucleotide polymorphisms (SNPs) (T-786C: rs2070744 and G894T: rs1799983) and one tagging SNP (rs7830) were evaluated in our study, and we assessed their association with the risk of CHD. Compared with the rs7830 CC/AC genotypes, the eNOS rs7830 AA genotype showed a significantly increased risk of
\end{abstract}


CHD (adjusted odds radio $(\mathrm{OR})=1.45,95 \%$ confidence interval $(\mathrm{CI}$ $=1.13-1.85)$. A stratified analysis was performed and showed that the association between the rs7830 AA genotype and CHD risk was stronger in patients with perimembranous ventricular septal defects (adjusted $\mathrm{OR}=1.62,95 \% \mathrm{CI}=1.20-2.20$ ). Our results suggest that the eNOS rs 7830 polymorphism may contribute to the susceptibility of sporadic CHD in a Chinese population.

Key words: eNOS; Congenital heart disease; Polymorphism

\section{INTRODUCTION}

Congenital heart disease (CHD) is the most common type of birth defect and noninfectious disease. The prevalence of this disease is approximately 6-8 in 1000 live births (Hoffman and Kaplan, 2002) and 1 in 10 still-borns, accounting for $25 \%$ of all human congenital abnormalities. The majority of CHD cases with monogenic inheritance is associated with non-cardiac malformations, and thereby constitutes a syndromic form of CHD. In contrast, most cases of CHD occur sporadically and present as a non-syndromic form (Bruneau, 2008). Although considerable effort has been made to detect the cause of CHD, few risk factors have been identified to date. The sporadic form of CHD is traditionally explained by a multifactorial inheritance model, which involves a multitude of susceptibility genes (Olson, 2004). Therefore, more evidence of CHD susceptibility genes is needed, especially with respect to low-penetrance susceptibility genes (common variants).

$\mathrm{NO}$ is a universal signaling molecule that is synthesized from L-arginine by a family of NO synthase (NOS) enzymes. There are at least 3 isoforms of NOS: neural NOS (nNOS), inducible NOS (iNOS), and endothelial NOS (eNOS) (Bloch et al., 1999). The endothelial isoform was originally identified in endothelial cells. eNOS is prominently expressed during the early stages of cardiogenesis, and plays an important role in normal heart development (Bloch et al., 1999; Feng et al., 2002).

The human eNOS gene is located on chromosome 7q35-q36 and contains 26 exons with a full length of $21 \mathrm{~kb}$. The $e N O S / \mathrm{T}-786 \mathrm{C}$ (rs2070744) variant is located in the 5'-flanking region and is suggested to be essential for promoter activity (Karantzoulis-Fegaras et al., 1999). Using a reporter gene, Miyamoto et al. (2000) revealed that the T/C substitution in position -786 in the promoter region reduced the transcription activity of eNOS by approximately $50 \%$, which was suggested to be due to the change in the binding activity of protein A1. Another polymorphism, G894T (rs1799983), in exon 7 of eNOS corresponds to a Glu/Asp substitution (Glu298Asp). The eNOS G894T polymorphism is associated with reduced basal NO production (Veldman et al., 2002). Senthil et al. (2005) demonstrated that endothelial cells exhibited genotype-dependent eNOS expression and enzyme activity for the eNOS G894T variant in an in vitro cell culture model. The polymorphism rs 7830 is situated within intron 23. It was speculated that this transversion might lead to aberrant splicing of the primary eNOS gene transcript (Gluba et al., 2009). Therefore, we selected two potentially functional single nucleotide polymorphisms (SNPs) (rs2070744, rs 1799983) and one tagging SNP (rs7830) of eNOS to evaluate the relationship between eNOS polymorphisms and CHD in Chinese subjects. 


\section{MATERIAL AND METHODS}

\section{Study population}

This study was performed under full compliance with all governmental policies and the Helsinki Declaration. All blood samples were obtained legally from the Affiliated Hospitals of Nanjing Medical University (NJMU), and were used only for research purposes. Since the participants were children, written informed consent was obtained from their next of kin or guardians. The protocol and consent forms were approved by the Institutional Review Board of NJMU, Nanjing, China. Nine hundred and forty-five affected children with sporadic CHD, confirmed by cardiac surgery, and 972 unrelated healthy individuals were included in this case-control study. Subjects for the study were recruited from the Affiliated Nanjing Children's Hospital of Nanjing Medical University, Nanjing, China, between March 2009 and December 2011. Potential study subjects were first surveyed at the clinics using a short questionnaire to determine their willingness to participate in a research study, and then demographic information was obtained through a face-to-face interview. After the interview, $4 \mathrm{~mL}$ venous blood was collected from each patient or control subject. Patients with other congenital disorders or known chromosomal abnormalities were excluded from the study. Exclusion criteria for mothers included maternal diabetes mellitus, phenyl ketonuria, maternal teratogen exposure, or maternal therapeutic drug exposure during the intrauterine period. These exclusion criteria were necessary since all of these factors can increase the risk of congenital anomalies. Control subjects were non-CHD outpatients from the same geographic area, who were age- and gender-matched to the CHD patients. They were recruited from the same hospital during the same period, and most had a diagnosis of trauma or infection. Controls with any congenital anomalies were excluded. All subjects were genetically unrelated ethnic Han Chinese.

\section{Genotyping}

Genomic DNA was extracted from peripheral blood leukocytes by using the DNA Mini Kit (QIAGEN, NED). Genotyping was performed using the multiplex SNaPshot technique. For rs1799983, the polymerase chain reaction (PCR) primers were 5'-AGATGAAGG CAGGAGACAGTG-3' (forward) and 5'-TCAATCCCTTTGGTGCTCACG-3' (reverse). For rs2070744, the PCR primers were 5'-TGGGAACTGTAGTTTCCCTAG-3' (forward) and 5'-AATTTCCTGGAACCCCCACAC-3' (reverse). For rs7830, the PCR primers were 5'-TGCCCTCTCTTTCCTATTTCC-3' (forward) and 5'-CAAAGGGGACCTGATGGAGTG -3 ' (reverse). The purified extension products were run on an ABI3130XL system according to manufacturer instructions (Applied Biosystems, Foster City, CA, USA). The results were analyzed using GeneMapper 4.0 (Applied Biosystems). Approximately 10\% of the samples were randomly selected for confirmation, and the results were $100 \%$ concordant.

\section{Statistical analysis}

Differences between the CHD patients and control subjects in the distributions of demographic characteristics, selected variables, and frequencies of genotypes of the three polymorphisms were evaluated using the Student $t$-test (for continuous variables) or the $\chi^{2}$ test (for 
categorical variables). The associations between the genotypes and risk of CHD were estimated by computing odds ratios (ORs) and their $95 \%$ confidence intervals (CIs) from unconditional logistic regression analysis, with adjustment for possible confounders. The genotype frequencies of these three polymorphisms among controls were all in agreement with Hardy-Weinberg equilibrium ( $\mathrm{P}=0.074$ for T-786C; $\mathrm{P}=0.505$ for $\mathrm{G} 894 \mathrm{~T} ; \mathrm{P}=0.115$ for $\mathrm{rs} 7830$ ). We controlled for the potential influence of multiple comparisons using the false-discovery rate method. $\mathrm{P}<$ 0.05 was considered to be statistically significant, and all statistical tests were two-sided. All statistical analyses were performed using the SAS 9.1.3 software (SAS Institute, Cary, NC, USA).

\section{RESULTS}

\section{Characteristics of the study group}

The characteristics of the CHD patients and control subjects included in the analysis are summarized in Table 1. We found no statistically significant differences between the CHD patients and controls in age or gender $(\mathrm{P}=0.606$ for age and $\mathrm{P}=0.558$ for gender $)$. Among the 945 CHD patients, 195 had cyanotic heart disease, 645 were diagnosed with a septation defect, 57 had patent ductus arteriosus (PDA), and 25 had a left-sided outflow obstruction defect.

\begin{tabular}{|c|c|c|c|c|c|}
\hline \multirow[t]{2}{*}{ Variables } & \multicolumn{2}{|c|}{ Cases } & \multicolumn{2}{|c|}{ Controls } & \multirow[t]{2}{*}{$\overline{\mathrm{Pa}^{\mathrm{a}}}$} \\
\hline & $\mathrm{N}$ & $\%$ & $\mathrm{~N}$ & $\%$ & \\
\hline Age [years (mean $\pm \mathrm{SD})]$ & \multicolumn{2}{|c|}{$2.87 \pm 0.18$} & \multicolumn{2}{|c|}{$2.94 \pm 0.17$} & 0.606 \\
\hline \multicolumn{6}{|l|}{ Gender } \\
\hline Male & 529 & 56.0 & 557 & 57.3 & 0.558 \\
\hline Female & 416 & 44.0 & 415 & 42.7 & \\
\hline \multicolumn{6}{|l|}{ CHD classification I } \\
\hline Cyanotic heart disease & 195 & 20.6 & & & \\
\hline Left-side obstruction defects & 25 & 2.7 & & & \\
\hline Septation defects & 645 & 68.3 & & & \\
\hline Patent ductus arteriosus & 57 & 5.5 & & & \\
\hline Other complex abnormality & 28 & 3.0 & & & \\
\hline \multicolumn{6}{|l|}{ CHD classification II } \\
\hline Septa and valve abnormalities only & 701 & 74.2 & & & \\
\hline Other CHD abnormalities & 244 & 25.8 & & & \\
\hline \multicolumn{6}{|l|}{ CHD classification III } \\
\hline Isolate CHD & 559 & 59.2 & & & \\
\hline Non-isolate CHD & 386 & 40.8 & & & \\
\hline
\end{tabular}

${ }^{\mathrm{a}}$ Two-sided $t$-test for age and $\chi^{2}$ test for the distributions of gender.

\section{Associations between $e N O S$ polymorphisms and risk of CHD}

The observed genotypes and allele frequencies for CHD and controls are shown in Table 2. The distributions of eNOS rs 7830 genotypes differed significantly between CHD and controls $\left(\mathrm{P}=0.012, \mathrm{P}_{\text {trend }}=0.016\right)$. Logistic regression analysis revealed that the $e N O S$ rs 7830 AA genotype, but not the AC genotype, was associated with a significantly increased risk of CHD compared with the $\mathrm{CC}$ genotype $(\mathrm{P}=0.005$, adjusted $\mathrm{OR}=1.48,95 \% \mathrm{CI}=1.12-1.94)$. Furthermore, in a recessive model, the eNOS rs7830 AA genotype was significantly associated with an increased risk for CHD compared to the rs $7830 \mathrm{CC} / \mathrm{AC}$ genotype (adjusted $\mathrm{OR}=1.45$, 
$95 \% \mathrm{CI}=1.13-1.85)$. However, the distributions of the eNOS G894T and T-786C genotypes were not significantly different between CHD patients and controls.

Table 2. Association of the eNOS polymorphisms with risk of CHD in a Chinese population.

\begin{tabular}{|c|c|c|c|c|c|c|}
\hline \multirow[t]{2}{*}{ Genotypes } & \multicolumn{2}{|c|}{ Cases $(\mathrm{N}=945)$} & \multicolumn{2}{|c|}{ Controls $(\mathrm{N}=972)$} & \multirow[t]{2}{*}{$\mathrm{P}^{\mathrm{a}}$} & \multirow[t]{2}{*}{${ }^{\mathrm{b}}$ Adjusted OR $(95 \% \mathrm{CI})$} \\
\hline & $\mathrm{N}$ & $\%$ & $\mathrm{~N}$ & $\%$ & & \\
\hline \multicolumn{7}{|l|}{ rs1799983 } \\
\hline GG & 759 & 80.3 & 757 & 77.9 & 0.311 & 1.00 (reference) \\
\hline TG & 173 & 18.3 & 204 & 21.0 & 0.147 & $0.84(0.67-1.06)$ \\
\hline TT & 13 & 1.4 & 11 & 1.1 & 0.690 & $1.13(0.50-2.55)$ \\
\hline $\mathrm{G}$ allele & 1691 & 89.5 & 1718 & 88.4 & 0.279 & \\
\hline $\mathrm{T}$ allele & 199 & 10.5 & 226 & 11.6 & & \\
\hline Trend test & & & & & 0.280 & \\
\hline \multicolumn{7}{|l|}{ rs2070744 } \\
\hline TT & 753 & 79.7 & 791 & 81.4 & 0.555 & 1.00 (reference) \\
\hline $\mathrm{TC}$ & 186 & 19.7 & 177 & 18.2 & 0.397 & $1.11(0.88-1.39)$ \\
\hline $\mathrm{CC}$ & 6 & 0.6 & 4 & 0.4 & 0.479 & $1.63(0.45-5.79)$ \\
\hline $\mathrm{T}$ allele & 1692 & 89.5 & 1759 & 90.5 & 0.322 & \\
\hline $\mathrm{C}$ allele & 198 & 10.5 & 185 & 9.5 & & \\
\hline Trend test & & & & & 0.309 & \\
\hline \multicolumn{7}{|l|}{ rs 7830} \\
\hline $\mathrm{CC}$ & 325 & 34.4 & 360 & 37.04 & 0.012 & 1.00 (reference) \\
\hline $\mathrm{AC}$ & 447 & 47.3 & 482 & 49.59 & 0.789 & $1.03(0.84-1.25)$ \\
\hline AA & 173 & 18.3 & 130 & 13.37 & 0.005 & $1.48(1.12-1.94)$ \\
\hline $\mathrm{AA} / \mathrm{CC}+\mathrm{AC}$ & 772 & 81.7 & 842 & 86.63 & 0.003 & $1.45(1.13-1.85)$ \\
\hline $\mathrm{C}$ allele & 1097 & 58.04 & 1202 & 61.83 & 0.017 & \\
\hline A allele & 793 & 41.96 & 742 & 38.17 & & \\
\hline Trend test & & & & & 0.016 & \\
\hline
\end{tabular}

a Two-sided $\chi^{2}$ test for the distributions of genotype and allele frequencies. ${ }^{\mathrm{b}}$ Adjusted for age and gender in logistic regression model.

\section{Stratified analysis of the eNOS rs7830 polymorphism and the risk of CHD}

We next evaluated the effect of the rs7830 polymorphism on CHD risk after the patients were stratified by specific CHD phenotypes. Since several patients had two or more types of cardiac defects, we chose only subjects showing a single phenotype. As shown in Table 3, we observed an increased risk of perimembranous ventricular septic defect (VSD) in individuals with the AA genotype (adjusted $\mathrm{OR}=1.62,95 \% \mathrm{CI}=1.20-2.20$ ). However, we found no association between the AA genotype and the risk of atrial septal defect (ASD), PDA, or tetralogy of Fallot (TOF). We also performed the same analysis for the eNOS T-786C and G894T polymorphisms, but no positive results were observed (data not shown).

Table 3. Stratification analyses between the eNOS genotypes and risk of CHD.

\begin{tabular}{|c|c|c|c|c|c|c|c|}
\hline \multirow[t]{3}{*}{ Phenotypes of CHD } & \multicolumn{4}{|c|}{ eNOS genotypes (case/control) } & \multirow[t]{3}{*}{$\mathrm{P}^{\mathrm{a}}$} & \multirow[t]{3}{*}{ Crude OR $(95 \% \mathrm{CI})$} & \multirow[t]{3}{*}{${ }^{\mathrm{b}}$ Adjusted OR $(95 \% \mathrm{CI})$} \\
\hline & \multicolumn{2}{|c|}{ AA genotype } & \multicolumn{2}{|c|}{$\mathrm{CC}+\mathrm{AC}$ genotypes } & & & \\
\hline & $\mathrm{N}$ & $\%$ & $\mathrm{~N}$ & $\%$ & & & \\
\hline Tetralogy of Fallot & $8 / 130$ & $12.3 / 13.4$ & $57 / 842$ & $87.7 / 86.6$ & 0.806 & $0.91(0.42-1.95)$ & $0.89(0.41-1.93)$ \\
\hline Perimembranous VSD & $85 / 130$ & $20.1 / 13.4$ & $338 / 842$ & $79.9 / 86.6$ & 0.001 & $1.63(1.21-2.20)$ & $1.62(1.20-2.20)$ \\
\hline ASD & $11 / 130$ & $19.3 / 13.4$ & $46 / 842$ & $80.7 / 86.6$ & 0.206 & $1.55(0.78-3.06)$ & $1.51(0.76-3.00)$ \\
\hline PDA & $9 / 130$ & $10.6 / 13.4$ & 76/842 & $89.4 / 86.6$ & 0.466 & $0.77(0.37-1.57)$ & $0.99(0.37-1.57)$ \\
\hline
\end{tabular}

${ }^{\mathrm{a}}$ Two-sided $\chi^{2}$ test for distribution between the cases and controls. ${ }^{\mathrm{b}}$ Adjusted for age and gender in logistic regression model. $\mathrm{VSD}=$ ventricular septic defect; $\mathrm{ASD}=$ atrial septal defect; $\mathrm{PDA}=$ patent ductus arteriosus. 


\section{DISCUSSION}

In this study, we observed that a significantly increased risk of CHD was associated with $e N O S$ (rs7830) polymorphisms. When we evaluated the effect of the eNOS genotypes on CHD risk after the patients were stratified by specific CHD phenotypes, we found that the risk of VSD was significantly increased for individuals carrying the AA genotype compared with the A allele. These findings suggested that polymorphisms of the eNOS gene may contribute to the susceptibility of sporadic CHD. To our knowledge, this is the first report to evaluate the association of the eNOS rs7830 polymorphism with CHD risk in a Chinese population.

The eNOS enzyme is responsible for the production of NO, which modulates homocysteine concentrations by inhibition of 5-methyltetrahydrofolate-homocysteine methyltransferase, the enzyme that synthesizes methionine from homocysteine and 5-methyltetrahydrofolate. Several heart defects were observed in two eNOS knockout mouse models, including septal defects and bicuspid aortic valves (Lee et al., 2000; Feng et al., 2002). In humans, some eNOS polymorphisms are associated with alterations in basal NO production. With respect to eNOS G894T polymorphisms, Shaw et al. (2005) observed a relationship between the embryonic eNOS 894T allele and a higher risk of conotruncal CHD in children. van Beynum et al. (2008) indicated that the eNOS G894T polymorphism was associated with increased risk of structural heart defects. Miyamoto et al. (2000) observed a 50\% reduction in the eNOS transcription rate associated with the $\mathrm{T} / \mathrm{C}$ substitution in position -786 of the promoter region. However, in the present study, no significant differences in the frequencies of genotypes or alleles of the G894T or T-786C polymorphisms were observed between CHD patients and healthy individuals. The discrepancies in the results of the present study and those of other studies might reflect differences in disease etiology and ethnic populations.

Polymorphism rs 7830 is located in intron 23 of the eNOS gene. There are only a few studies addressing this polymorphism, and most have detected an influence on the risks of renal disease, hypertension, or glaucoma (Li et al., 2011; Liao et al., 2011). In our study, we observed that the eNOS/rs7830 polymorphism might increase the risk of sporadic CHD in the Chinese Han population. It was speculated that this transversion might lead to aberrant splicing of the primary eNOS gene transcript based on the fact that a 5-nucleotide motif was generated that was identical to the original splice site. We next evaluated the effects of rs7830 genotypes on the risk of specific CHD phenotypes. For this analysis, we selected four subgroups: TOF, perimembranous VSD, ASD, and PDA. We found that the AA genotype was strongly associated with perimembranous VSD, but not PDA, TOF, or ASD. Congenital septal defects were also observed in an eNOS knockout mouse model. This may be attributed to the lower NO levels generated by eNOS locally, which may disturb the formation of heart septation. However, we did not find any association between the eNOS/rs7830 polymorphism and ASD. This could be attributed to the small sample size of isolated ASD patients.

Since we evaluated a hospital-based population, we could not avoid the possibility of selection bias of subjects that may have been associated with a particular genotype. Due to the low incidence of some phenotypes of CHD, we only chose four major phenotypes of CHD for our analysis of subgroups. For some rare phenotypes, the sample size was too small for an effective genetic association study. Therefore, our analysis of subgroups could not confirm the results for these anomalies. Since our results were based on significant associations between eNOS polymorphisms and the risk of CHD, the results do not establish a causal relationship for each phenotype of CHD, and should therefore be interpreted with caution. To validate our 
findings, additional functional studies are needed. Since our study was only conducted in a Chinese population, the results should be extrapolated to other ethnic groups cautiously.

\section{CONCLUSIONS}

In conclusion, we observed that a significantly increased risk of CHD was associated with the eNOS rs7830 polymorphism. Furthermore, we found that the association between genotypes of rs7830 and CHD risk was stronger in VSD subjects. Our results suggest that polymorphisms of eNOS may contribute to the susceptibility of sporadic CHD in the Chinese population. More detailed information about environmental exposure and gene-gene interactions are required to validate our findings.

\section{ACKNOWLEDGMENTS}

We are especially grateful to the patients and their parents for participating in this study, and to Dr. Bo Qian (The Affiliated Children's Hospital of Nanjing Medical University) for blood sample collection. Research supported by the National Nature Science Foundation of China (\#81070137) and the "333” Foundation for the Academic Leader of Jiangsu Province (Xuming Mo).

\section{REFERENCES}

Bloch W, Fleischmann BK, Lorke DE, Andressen C, et al. (1999). Nitric oxide synthase expression and role during cardiomyogenesis. Cardiovasc. Res. 43: 675-684.

Bruneau BG (2008). The developmental genetics of congenital heart disease. Nature 451: 943-948.

Feng Q, Song W, Lu X, Hamilton JA, et al. (2002). Development of heart failure and congenital septal defects in mice lacking endothelial nitric oxide synthase. Circulation 106: 873-879.

Gluba A, Banach M, Rysz J, Piotrowski G, et al. (2009). Is polymorphism within eNOS gene associated with the late onset of myocardial infarction? A pilot study. Angiology 60: 588-595.

Hoffman JI and Kaplan S (2002). The incidence of congenital heart disease. J. Am. Coll. Cardiol. 39: 1890-1900.

Karantzoulis-Fegaras F, Antoniou H, Lai SL, Kulkarni G, et al. (1999). Characterization of the human endothelial nitricoxide synthase promoter. J. Biol. Chem. 274: 3076-3093.

Lee TC, Zhao YD, Courtman DW and Stewart DJ (2000). Abnormal aortic valve development in mice lacking endothelial nitric oxide synthase. Circulation 101: 2345-2348.

Li J, Cun Y, Tang WR, Wang Y, et al. (2011). Association of eNOS gene polymorphisms with essential hypertension in the Han population in southwestern China. Genet. Mol. Res. 10: 2202-2212.

Liao Q, Wang DH and Sun HJ (2011). Association of genetic polymorphisms of eNOS with glaucoma. Mol. Vis. 17: 153-158.

Miyamoto Y, Saito Y, Nakayama M, Shimasaki Y, et al. (2000). Replication protein A1 reduces transcription of the endothelial nitric oxide synthase gene containing a $-786 \mathrm{~T}->\mathrm{C}$ mutation associated with coronary spastic angina. Hum. Mol. Genet. 9: 2629-2637.

Olson EN (2004). A decade of discoveries in cardiac biology. Nat. Med. 10: 467-474.

Senthil D, Raveendran M, Shen YH, Utama B, et al. (2005). Genotype-dependent expression of endothelial nitric oxide synthase (eNOS) and its regulatory proteins in cultured endothelial cells. DNA Cell Biol. 24: 218-224.

Shaw GM, Iovannisci DM, Yang W, Finnell RH, et al. (2005). Risks of human conotruncal heart defects associated with 32 single nucleotide polymorphisms of selected cardiovascular disease-related genes. Am. J. Med. Genet. A 138: 21-26.

van Beynum IM, Mooij C, Kapusta L, Heil S, et al. (2008). Common 894G>T single nucleotide polymorphism in the gene coding for endothelial nitric oxide synthase (eNOS) and risk of congenital heart defects. Clin. Chem. Lab. Med. 46: 1369-1375.

Veldman BA, Spiering W, Doevendans PA, Vervoort G, et al. (2002). The Glu298Asp polymorphism of the NOS 3 gene as a determinant of the baseline production of nitric oxide. J. Hypertens 20: 2023-2027. 